\title{
Extraperitoneal Urinary Bladder Rupture without Pelvic Fracture
}

\author{
Amiraj Singh, Satish Dalal, Anoop Yadav, Sethu Raman \\ Department of Surgery, Pt. B. D. Sharma Post Graduate Institute of Medical Sciences, Rohtak, Haryana, India.
}

\section{Corresponding Author:}

Dr. Amiraj Singh

Email id: amirajsingh92@gmail.com

This is an Open Access article distributed under the terms of the Creative Commons Attribution License (creativecommons.org/ licenses/by/3.0).

$\begin{array}{lll}\text { Received } & : & \text { January 7, } 2020 \\ \text { Accepted } & : & \text { April 24, } 2020 \\ \text { Published } & : & \text { May 30, } 2020\end{array}$

\begin{abstract}
Background: The extra-peritoneal bladder rupture without a pelvic fracture is uncommon and rarely reported. Case Report: A 9 years old male child presented with pain abdomen and an external wound over inguinal region secondary to abdominal trauma by the bicycle handle. Patient was having stable vitals and urine was seeping out of external wound. Ultrasound showed a small rent in urinary bladder and CECT findings were conclusive of extra-peritoneal bladder rupture with no pelvic girdle disruption. Patient was managed by conservative treatment with bladder drainage by Foley's catheter. Conclusion: The case is being reported to highlight the fact that bladder injury in children can be caused by trivial trauma.
\end{abstract}

Keywords: Abdominal Injuries, Drainage, Pain, Pelvic Bone, Rupture, Urinary Bladder Diseases.

\section{Introduction}

Urinary bladder injuries are relatively less frequent in the patients presenting with traumatic injury. In relation with other organs in blunt trauma injuries, its deep seated position in the bony pelvis protects it [1]. Not more than $2 \%$ of abdominal injuries comprise bladder injury requiring any surgical intervention [2]. Therefore bladder injury signifies high severity of trauma.

A rupture of urinary bladder secondary to injury can present as intra-peritoneal urine leak, extra-peritoneal leak or a combination of the aforesaid. In literature, the fracture of anterior pelvic arch associated with a bladder rupture is a well known entity due to its location [3]. Several reports have also shown rupture without any pelvic fracture. However, they exclusively belonged to intra-peritoneal leakage of urine. The extraperitoneal rupture without a pelvic fracture is rarely reported.

\section{Case Report}

A 9 year old male child presented to emergency department with history of fall from bicycle and suffered injury from the handle of bicycle. Patient presented to hospital after 24 hours of injury and was having chief complaints of pain abdomen and an external wound over inguinal region. He was well oriented to time, place and person with GCS 15/15. On general physical examination, he was having tachycardia; pulse rate $110 / \mathrm{min}$, blood pressure $114 / 70 \mathrm{mmHg}$ with other vitals being stable. On systemic examination, there was tenderness present over lower abdomen which was localized to hypogastrium. Local examination revealed a swelling present at left inguinoscrotal region and a small external wound over left inguinal region from which clear fluid was seeping out. The fluid seemed to be urine as it was pale yellow in colour and of ammoniacal smell. There was no blood at urethral meatus and no history of hematuria. Initial resuscitation of the patient according to 
institutional protocol was done and routine blood investigations were sent. Hemoglobin was 10.4 $\mathrm{gm} / \mathrm{dL}$ and TLC were 8000/hpf. Biochemical investigations revealed blood urea $28 \mathrm{mg} / \mathrm{dL}$ and serum creatinine $0.7 \mathrm{mg} / \mathrm{dL}$.

Ultrasonography of the abdomen showed a rent in anterolateral surface of urinary bladder [Fig.1]. All other visualized viscera and solid organs were normal with no intra-peritoneal collection. Contrast enhanced computed tomography revealed discontinuation of the antero-inferior urinary bladder wall from where urine was seeping out to external skin. There was neither any evidence of pelvic bone fracture nor intra-peritoneal collection [Fig.2]. Patient was managed conservatively by intravenous antibiotics and Foley's catheter (FG10) insertion. Daily vitals and urine output was monitored. Patient got discharged after five days of hospital stay with Foley's catheter in situ and was advised for follow up after 2 weeks. A CT cystogram was done at three weeks which showed complete healing and no residual rent/leak in the urinary bladder. Foley's catheter was removed after three weeks, when patient came for follow up. Child remained well thereafter.

\section{Discussion}

The urinary bladder is a muscular sac in the pelvis which stores urine, allowing urination to be infrequent and controlled. It lies deep in the bony pelvis above and behind the pubic symphysis. The neck of the bladder is fixed to surrounding structures by reflections of the visceral pelvic fascia, and by the true and false ligaments of the pelvis. Besides this, in males, there is an attachment to the pubis by the puboprostatic ligament. The bladder receives further support from the urogenital diaphragm and obturator internus muscles in its lateral and inferior side. The bladder is covered by the peritoneum at the dome postero-superiorly. Elsewhere it is surrounded by a layer of loose extra-peritoneal areolar connective tissue which separates it from the anterior abdominal wall i.e. space of Retzius

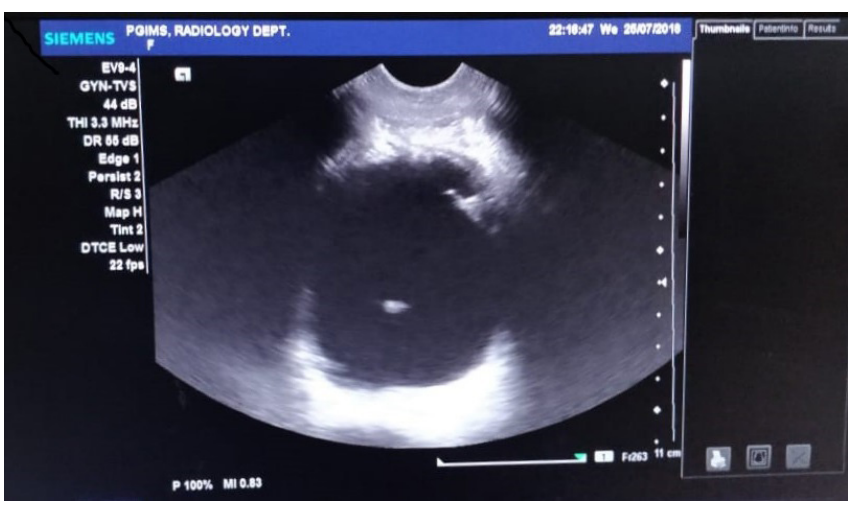

Fig.1: USG of urinary bladder showing rent in antero-lateral surface.

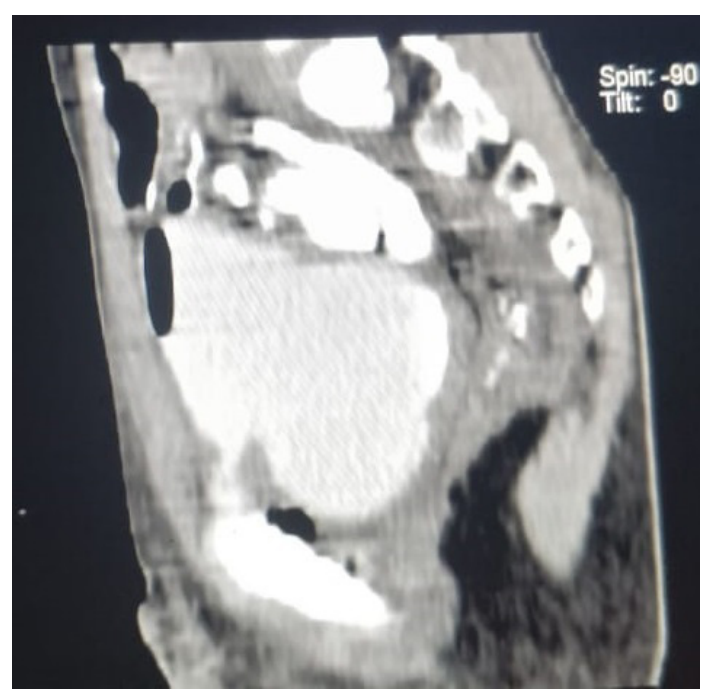

Fig.2: Para-saggital view of CECT abdomen showing rent in antero-inferior surface of urinary bladder wall.

anteriorly, and posteriorly it separates from the rectum in men while the uterus and cervix in women $[4,5]$. Age is also an important consideration. The bladder in children has a less protected and more intra-abdominal position. It descends into the pelvis usually by the age 20 years $[6,7]$.

Urinary bladder injuries are caused by a high energy blunt trauma to abdomen that disrupts the bony pelvis, a direct blow to a distended bladder, penetrating injuries or iatrogenic causes. When bladder ruptures, urine leaks intra-peritoneally (38$40 \%$ ), extra-peritoneally (54-56\%) or combination of both $(5-8 \%)$ depending on nature/mechanism 
of trauma, volume of urine contained while trauma or any pre-existing pathological conditions of pelvis [8]. Intra-peritoneal bladder rupture occurs when there is a sudden rise in intra-vesical pressure secondary to a blow to the pelvis or lower abdomen. This increase in pressure leads to rupture of the dome which is the weakest and most mobile part of the bladder [8]. It is known to occur with or without pelvic fracture. The extra-peritoneal bladder ruptures are seen almost exclusively with pelvic fractures. The bladder is usually sheared on the antero-lateral wall near the bladder base by disruption of ligamentous attachment between the bladder and pelvis in conjunction with pelvic girdle disruption. Less commonly, the bladder is lacerated by a sharp bony spicule. Urinary extravasation may extend to thigh, scrotum, anterior abdominal wall and retroperitoneal space [8].

Thus there are two mechanisms of injury which can cause bladder rupture i.e disruption of the pelvic girdle leading to extra-peritoneal rupture or compression of the full bladder with a resultant intra-peritoneal rupture. Extra-peritoneal bladder rupture in the absence of disruption of pelvic girdle is very uncommon entity which we had seen in our case. Therefore, we must conjuncture that an extra-peritoneal rupture can occur in a fashion cognate to an intra-peritoneal rupture. This pattern of injury can be secondary to some local factors or severity of trauma. Also, there can be a possibility that pelvic fracture is mere a coincidental finding instead of a causative factor of urinary bladder rupture. Moreover, in children since bladder is being an intra-abdominal organ partly, the chances of injury are more even by a trivial trauma.

\section{Conclusion}

Even a trivial trauma can lead to serious injuries like extra-peritoneal bladder rupture in children. There may be some unknown factors and mechanisms responsible for extra-peritoneal rupture of bladder besides pelvic fracture.

Contributors: AS: manuscript writing, literature review and surgery; SD, AY: critical inputs into the manuscript and surgery; SR: literature review, discussion and surgery. AS will act as a study guarantor. All authors approved the final version of this manuscript and are responsible for all aspects of the study.

Funding: None; Competing interests: None stated.

\section{References}

1. Carol PR, McAninch JW. Major bladder trauma: Mechanisms of injury and a unified method of diagnosis and repair. J Urol. 1984;132:254-257.

2. Cass AS. The multiple injured patient with bladder trauma. J Trauma. 1984;24:731-734.

3. Hayes EE, Sandler CM, Corriere JN. Management of the ruptured bladder secondary to blunt abdominal trauma. J Urol. 1983;129:946-948.

4. Lich R Jr., Howerton LW, Amin M. Anatomy and surgical approach to the urogenital tract in the male. In: Harrison JH (ed). Campbell's Urol. $4^{\text {th }}$ ed. W. B. Saunders Co:Walsh. Philadelphia. 1978; pp.20.

5. Clark SS, Prudencio RF. Lower urinary tract injuries associated with pelvic fractures. Diagnosis and management. Surg Clin North Am. 1972;52:183-201.

6. Merchant WC 3rd, Gibbons MD, Gonzales ET Jr. Trauma to the bladder neck, trigone and vagina in children. J Urol. 1984;131:747-750.

7. Hirsch S, McCally DS, Kandler C. The etiology, diagnosis, and treatment of bladder trauma. N Y State J Med. 1983;83:316-319.

8. Gomez RG, Ceballos L, Coburn M, Corriere JN, Dixon $\mathrm{CM}$, Lobel B, et al. Consensus statement on bladder injuries. BJU Int. 2004;94:27-32. 